\title{
BMJ Open Bone health in symptomatic carriers of haemophilia A: a protocol for a multicentre prospective matched- cohort study
}

Grace H Tang, ${ }^{1}$ Erin Norris, ${ }^{2}$ Jessica Petrucci, ${ }^{1}$ Paula D James, ${ }^{3}$ Adrienne Lee, ${ }^{4}$ Man-Chiu Poon, ${ }^{4}$ Georgina Floros, ${ }^{1}$ Laurence Boma-Fischer, ${ }^{1}$ Jerry Teitel, ${ }^{1}$ Rosane Nisenbaum, ${ }^{5,6}$ Michelle Sholzberg (i) ${ }^{1}$

\section{ABSTRACT}

To cite: Tang GH, Norris $\mathrm{E}$, Petrucci J, et al. Bone health in symptomatic carriers of haemophilia A: a protocol for a multicentre prospective matchedcohort study. BMJ Open 2019;9:e032891. doi:10.1136/ bmjopen-2019-032891

- Prepublication history for this paper is available online. To view these files, please visit the journal online (http://dx.doi org/10.1136/bmjopen-2019032891).

Received 11 July 2019 Revised 30 0ctober 2019 Accepted 11 November 2019

Check for updates

(C) Author(s) (or their employer(s)) 2019. Re-use permitted under CC BY-NC. No commercial re-use. See rights and permissions. Published by BMJ.

For numbered affiliations see end of article.

Correspondence to Dr Michelle Sholzberg; SholzbergM@smh.ca health.
Introduction Haemophilia A is an X linked inherited bleeding disorder, caused by a decrease in coagulation factor VIII. Persons with haemophilia experience repeated musculoskeletal bleeding, which can lead to decreased range of motion, irreversible joint damage, low bone mineral density (BMD), and are at greater risk for osteoporosis. Women heterozygous for this mutation, also known as haemophilia A carriers, can have bleeding symptoms and even experience joint bleeding evidenced by radiological soft tissue and osteochondral changes. The prevalence of low BMD as a risk factor for osteoporosis has never been evaluated in carriers of haemophilia, and given the recent findings which suggest subclinical musculoskeletal bleeding in carrier women, we hypothesise that they too are at risk of impaired bone

Methods and analysis This is a national multicentre prospective matched-cohort study to compare BMD T-scores among symptomatic haemophilia A carriers, 50 years of age or older, with age-matched and body mass index-matched non-carriers (1:1). A total of 40 symptomatic carriers and 40 matched non-carriers will be recruited from St. Michael's Hospital, Kingston General Hospital in Ontario, Canada and Foothills Medical Centre in Alberta, Canada. Multivariable linear regression models will be used to estimate the effect of haemophilia carriership on BMD T-scores, adjusting for age, body mass index and other relevant covariates.

Ethics and dissemination The protocol was designed and will be conducted in compliance with applicable laws, rules and regulations. Research ethics approval was obtained from St. Michael's Hospital, Foothills Medical Centre, and Kingston General Hospital. Findings will be presented at international venues such as the American Society of Haematology and the World Federation of Haemophilia World Congress. The authors of this study will seek publication in journals such as Blood, Journal of Thrombosis and Haemostasis, American Journal of Hematology and British Journal of Haematology.

\section{INTRODUCTION}

Haemophilia is an $\mathrm{X}$ linked inherited bleeding disorder, caused by a decrease in
Strengths and limitations of this study

- This will be the first study to evaluate bone health measured by bone mineral density T-scores among carriers of haemophilia $A$.

- This is a multicentre national matched-cohort study with a sample size of 40 haemophilia A carriers and 40 age-matched and body mass index-matched non-carriers.

- The small sample size may limit statistical power to conduct additional exploratory analyses.

- The restriction to women 50 years or older may limit generalisability to younger haemophilia A carriers.

coagulation factor VIII (haemophilia A). ${ }^{1}$ Bleeding in these patients typically occurs in joints and muscles, but bleeding at any site can occur. ${ }^{23}$ Repeated musculoskeletal bleeding can lead to decreased range of motion, muscle atrophy and irreversible joint damage. ${ }^{2}$ The exact pathophysiology of low bone mineral density (BMD) in haemophilia is yet to be elucidated. It may be possible that factor VIII itself is responsible for the maintenance of bone integrity. ${ }^{4}$ However, the underlying mechanism is likely multifactorial and due to recurrent bleeding in the musculoskeletal system with local inflammation, subsequent haemophilic arthropathy and limited weight-bearing physical activity. ${ }^{56}$

Several studies have identified a high prevalence of low BMD and increased risk of osteoporosis in patients with haemophilia..$^{5-10}$ Iorio et al pooled 101 adult cases and found a significant lower lumbar BMD in patients with severe haemophilia compared with controls (pooled standardised mean difference (SMD) $-1.379,95 \%$ CI -2.353 to $\left.-0.403, \mathrm{I}^{2}=87.9 \%\right){ }^{7}$ Another meta-analysis showed similar SMD values in lumbar BMD (SMD -0.56, 95\% CI -0.84 to $\left.-0.28, \mathrm{I}^{2}=51 \%\right){ }^{8}$ Furthermore, the 
authors demonstrated a reduced femoral neck BMD in adult patients with haemophilia compared with controls $\left(\mathrm{SMD}=-0.82,95 \%\right.$ CI -1.21 to $\left.0.44, \mathrm{I}^{2}=63 \%\right) .8$

Recent findings suggest that women who are heterozygous for factor VIII mutations, commonly known as haemophilia carriers, may also have significant bleeding symptoms and lower health-related quality of life (HRQoL). ${ }^{11}{ }^{12}$ In fact, $30 \%$ of haemophilia carriers have low coagulation factor levels and may experience excessive bleeding. ${ }^{13}$ A study conducted by Plug et al, reported that carriers with factor VIII activity levels between 0.05 and $0.60 \mathrm{IU}$ had prolonged bleeding from small wounds, and after tooth extraction, tonsillectomy and surgery. ${ }^{1}$ Another study conducted by Miesbach et al, showed that haemophilia A carriers with high factor VIII levels (up to $60 \%$ ) also experience increased risk of bleeding. ${ }^{13} \mathrm{~A}$ survey conducted by Paroskie et alfound that haemophilia A carriers self-reported a higher frequency of bleeding compared with normal controls. ${ }^{14}$ More recently, a study by James et al reported that haemophilia carriers had a higher mean bleeding score using the International Society on Thrombosis and Haemostasis Bleeding Assessment Tool (ISTH-BAT) compared with age-matched controls (5.7 vs $1.43, \mathrm{p}<0.0001)$ and that factor VIII activity correlates poorly with the ISTH-BAT score. ${ }^{15}$

In addition to increased bleeding tendencies and lower HR-QoL, Gilbert et al noted that haemophilia A carriers also experience subclinical joint bleeding which could lead to structural joint damage. ${ }^{16}$ This was demonstrated by soft tissue and osteochondral changes on joint MRI. ${ }^{16}$ Sidonio et al demonstrated that haemophilia carriers have a reduced mean joint range of motion compared with historical controls. ${ }^{17}$ Together, this suggests musculoskeletal bleeding among carriers.

A growing body of evidence suggests that bleeding tendencies in haemophilia carriers may be more similar to affected men than originally thought. The prevalence of low BMD as a risk factor for osteoporosis and fractures has never been evaluated in carriers of haemophilia, and given the recent findings which suggest subclinical musculoskeletal bleeding in carrier women, we hypothesise that they too are at risk of impaired bone health. We therefore propose a prospective cohort, multicentre study to compare BMD in adult perimenopausal symptomatic haemophilia A carriers with age-matched and body mass index (BMI)-matched non-carriers via dual-energy X-ray absorptiometry (DEXA).

\section{Study objectives}

Primary objective:

- To compare adult perimenopausal and postmenopausal ( $\geq 50$ years) symptomatic haemophilia A carriers with age-matched and BMI-matched noncarriers with respect to BMD T-scores measured by DEXA scans of the hip and spine.

The secondary objectives are:

- To compare the prevalence of low bone mass density (defined as a T-score between -1 and -2.5) in symptomatic haemophilia A carriers with age-matched and BMI-matched non-carriers.

- To compare the prevalence of osteoporosis (defined as T-score $\leq-2.5$ ) in symptomatic haemophilia A carriers with age-matched and BMI-matched non-carriers.

- To compare BMD scores between symptomatic haemophilia A carriers with age-matched and BMImatched non-carriers.

\section{METHODS AND ANALYSIS \\ Study design}

This is a national multicentre prospective matched cohort study to compare BMD T-scores among symptomatic haemophilia A carriers ('exposed') with age-matched and BMI-matched non-carriers ('unexposed'). This is an exploratory study as no studies have investigated this association in this patient population previously. See figure 1 for the study schema.

\section{Study population}

We are interested in symptomatic haemophilia A carriers during their perimenopausal and postmenopausal years,

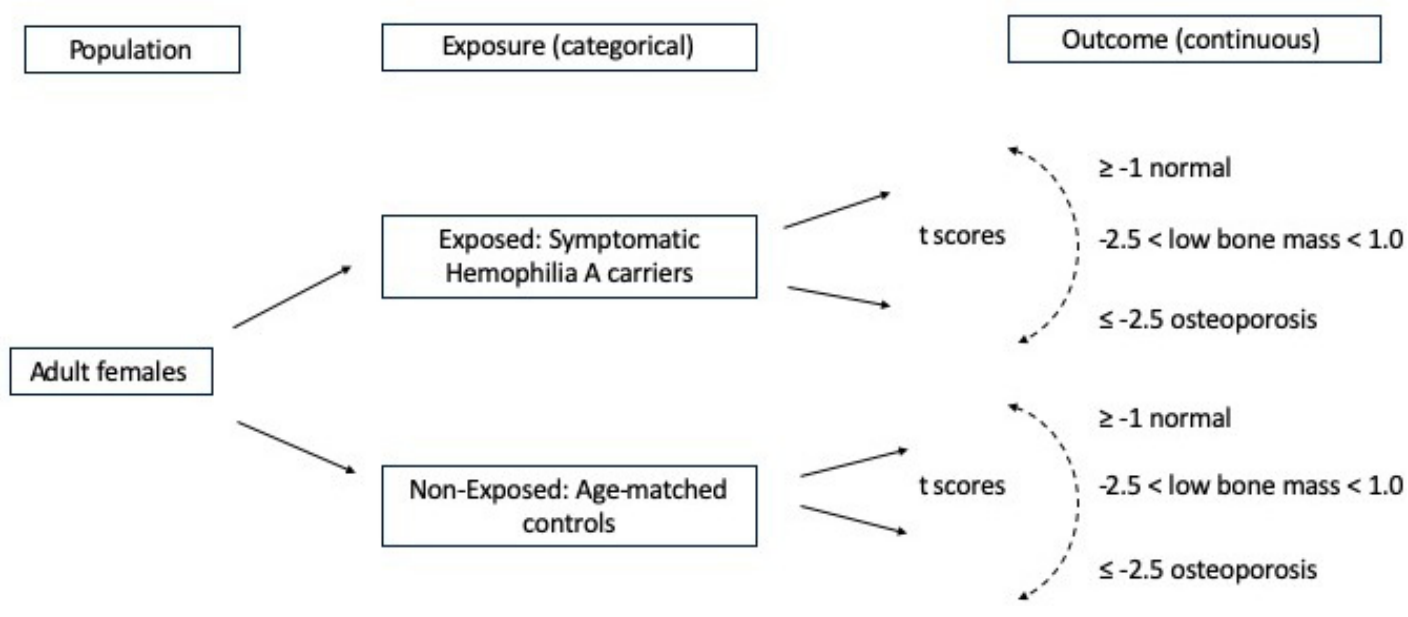

Figure 1 Study schema. 
Table 1 Inclusion and exclusion criteria for haemophilia a carriers

\section{Inclusion}

1. Symptomatic haemophilia A carriers defined as a SelfBAT Score $\geq 6$.

2. Symptomatic haemophilia A carriers $\geq 50$ years old.

3. (a) Confirmed diagnosis of carriership through genetic factor VIII sequencing.

(b) Or women who have appropriate family history (daughter of a man with haemophilia or mother of two sons with haemophilia or mother of one son with haemophilia and at least one other affected male relative).

All affected men must have a confirmed diagnosis through genetic screening factor VIII sequencing (from standard of care).

\section{Exclusion}

1. Under the age of 50 years.

2. Women who are pregnant or lactating.

3. Diagnosed with another bleeding disorder (eg, von Willebrand disease).

4. Participants with a previous diagnosis of hepatitis $\mathrm{C}$ virus (HCV) or HIV.

5. Participants with a history of: kidney failure, Cushing's disease, liver impairment, anorexia and/or bulimia, rheumatoid arthritis, obstructive pulmonary disease, scurvy.

6. Participants on bisphosphonate therapy, chronic steroid use, or anti-resorptive therapy.

Self-BAT, Self-Administered Bleeding Assessment Tool.

since the effect of our primary outcome of low BMD may not be evident in younger women. ${ }^{18}$ In Canada, the average age for menopause is 52 years, ranging from ages 42 years through 56 years. ${ }^{19}$ For this reason, we have limited the study eligibility to women 50 years of age or older. A symptomatic haemophilia carrier is defined as a woman with heterozygosity for a coagulation factor VIII mutation or appropriate and confirmed family history of haemophilia A, and a positive bleeding history (SelfAdministered Bleeding Assessment Tool (Self-BAT) score of 6 or more). While factor VIII activity is not being used in the definition of carriership, we plan to analyse BMD in relation to factor VIII levels. No more than two family members in each affected family will be enrolled in the study to minimise selection and confounding bias (ie, similar genetic background and predisposing risk factors for low BMD). All haemophilia A carriers will be age-matched to a non-carrier in a 1:1 ratio. Categorical age matching will be used within 5-year intervals. BMI matching will be categorised by underweight $(\mathrm{BMI}<18.5)$, normal (BMI 18.5-24.9) and overweight (BMI >25) as per the Canadian Guidelines for Body Weight Classification in Adults. ${ }^{20} \mathrm{BMI}$ is calculated as weight $(\mathrm{kg}) /$ height $\left.(\mathrm{m})^{2}\right) .{ }^{20}$
Matching participants by age and BMI is needed to minimise/eliminate the effect of these confounding variables on the association between haemophilia A carriership and low BMD, since older age and low BMI are well known risk factors for low BMD. See tables 1 and 2 for the inclusion and exclusion criteria for haemophilia A carriers and non-carriers. Participants with a previously established bleeding disorder will be excluded as we want to focus on the effect of haemophilia A carriership on bone density. Participants with a diagnosis of HIV, hepatitis $\mathrm{C}$ virus, history of kidney failure, Cushing's disease, liver impairment, anorexia and/or bulimia, rheumatoid arthritis, obstructive pulmonary disease, scurvy will also be excluded as these conditions are known risk factors for osteoporosis. Participants on bisphosphonate therapy, chronic steroid use, or antiresorptive therapy will be excluded as these therapies can affect BMD T-scores. One of the inclusion criteria for non-carriers will be factor VIII activity $>80 \%$, as levels below this might inadvertently lead to inclusion of subjects with undiagnosed von Willebrand disease or even haemophilia A carriership.

Table 2 Inclusion/exclusion criteria for non-carriers

\section{Inclusion}

1. Women who score $<6$ in the Self-BAT.

2. Women who are $\geq 50$ years old.

3. Negative family history of excessive bleeding, bleeding disorder and/or haemophilia.

4. Factor VIII levels $>80 \%$.

\section{Exclusion}

1. Under the age of 50 years.

2. Women who are pregnant or lactating.

3. Diagnosed with another bleeding disorder (eg, von Willebrand disease).

4. Participants with a previous diagnosis of HCV or HIV.

5. Participants with a history of kidney failure, Cushing's disease, liver impairment, anorexia and/or bulimia, rheumatoid arthritis, obstructive pulmonary disease, scurvy.

6. Participants on bisphosphonate therapy, chronic steroid use or antiresorptive therapy. 


\section{Sample size}

Due to the novelty of this study, a sample size could not be estimated based on previous literature. Therefore, we are choosing to use a convenient and cost-feasible sample size of 80 patients in total (40 haemophilia A carriers, 40 age-matched and BMI-matched non-carriers) for this hypothesis-generating study. Of note, 40 pairs achieve approximately $90 \%$ power to detect a T-score mean difference between the groups of $0.5 \mathrm{SD}$ when using a paired t-test at the $5 \%$ significance level.

\section{Patient and public involvement}

No patient was involved in the development of the protocol.

\section{Measurement of covariates}

\section{Self-Administered Bleeding Assessment Tool}

The Self-BAT will be completed by the participant and scored by the treating physician, study coordinator or research assistant at each respective centre. The Self-BAT asks questions regarding previous bleeding history and takes approximately $5-10 \mathrm{~min}$ to complete. ${ }^{21}$ The Self-BAT has been validated among haemophilia carriers (sensitivity $71 \%$, specificity $51 \%$, positive predictive value 0.51 and negative predictive value 0.71$).^{22}$

The Self-BAT was developed from the ISTH-BAT and translated to make the document comprehensible to people with grade IV level education. The scores for each question range from 0 to +4 . The overall bleeding score is summative and scores $\geq 6$ are considered abnormal. ${ }^{21} 22$

\section{Bone-Specific Physical Activity Questionnaire}

The Bone-Specific Physical Activity Questionnaire (BPAQ) is self-administered and designed to estimate levels of physical activity over an entire life span. ${ }^{21}$ Respondents record type, frequency and years of physical activity involvement. The BPAQ consists of three independent scores: the past period (pBPAQ, from 1 year of age to the current age), the current period (cBPAQ, previous 12 months) and the total period (average of pBPAQ and cBPAQ). Algorithms used to analyse BPAQ scores are available using the online calculator (www.fithdysign.com/BPAQ/) and described in detail by Weeks et $a l .{ }^{23}$ While the BPAQ is not validated in the haemophilia carrier population, the score will provide insight into the study participants' lifetime physical activity which may correlate with risk of diminished BMD. The BPAQ takes approximately 5-15 min to complete.

\section{Laboratory tests}

The following tests will be collected from all recruited participants: factor VIII clotting activity by one-stage assay, 25-OH vitamin D, complete blood count, von Willebrand factor antigen, von Willebrand factor ristocetin cofactor activity, and $\mathrm{ABO}$ blood group.

The von Willebrand factor antigen and von Willebrand factor ristocetin cofactor activity will be ordered to confirm the absence of von Willebrand disease in our study population. ABO blood group and 25-OH vitamin $\mathrm{D}$ will be collected for demographic purposes.

\section{Measurement of outcome}

BMD T-scores are the primary outcome and will be calculated for all study participants, providing a comparison between haemophilia carriers and the age-matched and BMI-matched non-carriers (primary outcome). BMD will only be assessed on the hip and spine. Z-scores which are adjusted for age, sex, weight, and ethnic or racial origin are not used since we are evaluating women who are over the age of 50 years and postmenopausal with a matched non-carrier cohort. T-scores are chosen as they are clinically validated in women age 50 years and over, and is appropriate for our outcome assessment.

\section{Statistical analysis plan}

Descriptive statistics (mean, SD, median, IQR, proportions) will be used to summarise patient demographics. Box plots and histograms will illustrate sample outcomes and characteristics distribution by selected patient factors. The association between categorical variables will be tested using $\chi^{2}$ or Fisher's exact tests. Correlations between continuous variables will be estimated by Pearson or Spearman correlation coefficients. Unadjusted analyses will employ the paired t-test to compare groups with respect to the primary outcome and other continuous variables. Multivariable linear regression models will be considered to estimate the effect of haemophilia carriership on BMD T-scores, adjusting for age and other potential relevant covariates. SAS V.9.4 (SAS Institute, Cary, North Carolina, USA) will be used to perform the analyses. Exploratory and a priori subgroup analyses for this study include:

1. To compare haemophilia carriers with historical nadir factor VIII levels $<50 \%$ with haemophilia carriers with historical factor VIII $\geq 50 \%$ with respect to their BMD T-scores.

2. To compare haemophilia carriers with a history of musculoskeletal bleeding compared with those without with respect to their BMD T-scores.

\section{Limitations}

There are some limitations to our study that are worthy of mention. Reporting and recall bias may occur with the Self-BAT, as questions are asked about previous bleeding episodes which may be impacted by a carrier's perspective given her exposure to an affected man with haemophilia A. We did not collect socioeconomic data, which may be a possible confounder for bone health (ie, diet and exercise). The small sample size may limit statistical power to conduct further exploratory analysis. Our eligibility criteria of women over age 50 years may exclude younger haemophilia carriers with low BMD scores.

\section{Conclusions}

While there is extensive literature on men with haemophilia, few studies have focused on bleeding and healthrelated consequences in haemophilia carriers. To our 
knowledge, this will be the first study to investigate the association of low BMD with haemophilia heterozygosity. We believe that carriers will have lower bone mass compared with non-carriers of similar age and if we are right, this could change the paradigm of clinical care offered to these women. Future modifications to carrierrelated care may include an earlier assessment of fracture risk, regular imaging to assess for subclinical joint bleeding and prophylactic intervention for carriers with musculoskeletal bleeding whether overt or subclinical.

\section{Author affiliations}

${ }^{1}$ Hematology/Oncology, St. Michael's Hospital, Toronto, Ontario, Canada

${ }^{2}$ Rheumatology, St. Michael's Hospital, Toronto, Ontario, Canada

${ }^{3}$ Hematology, Kingston General Hospital, Kingston, Ontario, Canada

${ }^{4}$ Hematology, University of Calgary, Calgary, Alberta, Canada

${ }^{5}$ Applied Health Research Centre, MAP Centre for Urban Health Solutions, St.

Michael's Hospital, Toronto, Ontario, Canada

${ }^{6}$ Dalla Lana School of Public Health, University of Toronto, Toronto, Ontario, Canada

Contributors Conception and design: GHT, MS. Development of methodology: GHT, EN, JP, PDJ, AL, M-CP, GF, LB-F, JT, RN, MS. Statistical Analysis: RN. Draft of protocol: GHT, MS. Review of protocol: GHT, EN, JP, PDJ, AL, M-CP, GF, LB-F, JT, RN, MS.

Funding This work is supported by the Canadian Hemophilia Society/Pfizer Care Until Care Grant.

Patient consent for publication Not required.

Provenance and peer review Not commissioned; externally peer reviewed.

Open access This is an open access article distributed in accordance with the Creative Commons Attribution Non Commercial (CC BY-NC 4.0) license, which permits others to distribute, remix, adapt, build upon this work non-commercially, and license their derivative works on different terms, provided the original work is properly cited, appropriate credit is given, any changes made indicated, and the use is non-commercial. See: http://creativecommons.org/licenses/by-nc/4.0/.

ORCID iD

Michelle Sholzberg http://orcid.org/0000-0003-1220-0301

\section{REFERENCES}

1 Plug I, Mauser-Bunschoten EP, Bröcker-Vriends AHJT, et al. Bleeding in carriers of hemophilia. Blood 2006;108:52-6.

2 Society $\mathrm{CH}$. The symptoms of hemophilia, 2016. Available: http:// www.hemophilia.ca/en/bleeding-disorders/hemophilia-a-and-b/thesymptoms-of-hemophilia/

3 Canadian Hemophilia Society. Symptomatic carriers of hemophilia. all about Hemoph, 2010. Available: www.hemophilia.ca
4 Recht M, Liel MS, Turner RT, et al. The bone disease associated with factor VIII deficiency in mice is secondary to increased bone resorption. Haemophilia 2013;19:908-12.

5 Anagnostis $\mathrm{P}$, Vakalopoulou S, Slavakis A, et al. Reduced bone mineral density in patients with haemophilia $A$ and $B$ in northern Greece. Thromb Haemost 2012;107:545-51.

6 Wells AJ, McLaughlin P, Simmonds JV, et al. A case-control study assessing bone mineral density in severe haemophilia $A$ in the UK. Haemophilia 2015;21:109-15.

7 Iorio A, Fabbriciani G, Marcucci M, et al. Bone mineral density in haemophilia patients: a meta-analysis. Thromb Haemost 2010;103:596-603.

8 Paschou SA, Anagnostis P, Karras S, et al. Bone mineral density in men and children with haemophilia A and B: a systematic review and meta-analysis. Osteoporos Int 2014;25:2399-407.

9 Kempton CL, Antoniucci DM, Rodriguez-Merchan EC. Bone health in persons with haemophilia. Haemophilia 2015;21:568-77.

10 Rodriguez-Merchan EC, Valentino LA. Increased bone resorption in hemophilia. Blood Rev 2019;33:6-10.

11 Gilbert L, Paroskie A, Gailani D, et al. Haemophilia a carriers experience reduced health-related quality of life. Haemophilia 2015;21:761-5.

12 Gillham A, Greyling B, Wessels T-M, et al. Uptake of genetic counseling, knowledge of bleeding risks and psychosocial impact in a South African cohort of female relatives of people with hemophilia. $J$ Genet Couns 2015;24:978-86.

13 Miesbach W, Alesci S, Geisen C, et al. Association between phenotype and genotype in carriers of haemophilia a. Haemophilia 2011;17:246-51.

14 Paroskie A, Oso O, Almassi B, et al. Both hemophilia health care providers and hemophilia a carriers report that carriers have excessive bleeding. J Pediatr Hematol Oncol 2014;36:e224-30.

15 James PD, Mahlangu J, Bidlingmaier C, et al. Evaluation of the utility of the ISTH-BAT in haemophilia carriers: a multinational study. Haemophilia 2016;22:912-8.

16 Gilbert L, Rollins L, Hilmes M, et al. Haemophilia a carriers demonstrate pathological and radiological evidence of structural joint changes. Haemophilia 2014;20:e426-9.

17 Sidonio R, Mili F, Tengguo L, et al. Females with FVIII and fix deficiency have reduced joint range of motion 2015;89:831-6.

18 Finkelstein JS, Brockwell SE, Mehta V, et al. Bone mineral density changes during the menopause transition in a multiethnic cohort of women. J Clin Endocrinol Metab 2008;93:861-8.

19 Canadian Women's Health Network. Menopause, 2012. Available: http://www.cwhn.ca/en/faq/menopause

20 Health Canada. Canadian Guidelines for Weight Classification in Adults - Quick Reference Tool for Professionals. Can Guidel Body Weight Classif Adults 2003.

21 Deforest M, Grabell J, Albert S, et al. Generation and optimization of the self-administered bleeding assessment tool and its validation as a screening test for von Willebrand disease. Haemophilia 2015;21:e384-8.

22 Young JE, Grabell J, Tuttle A, et al. Evaluation of the selfadministered bleeding assessment tool (Self-BAT) in haemophilia carriers and correlations with quality of life. Haemophilia 2017;23:e536-8.

23 Weeks BK, Beck BR. The BPAQ: a bone-specific physical activity assessment instrument. Osteoporos Int 2008;19:1567-77. 